\title{
Singularities, Normal Forms, and Motion Planning for Non-holonomic Robotic Systems
}

\author{
Krzysztof Tchon, Joanna Ratajczak \\ Department of Cybernetics and Robotics, Electronics Faculty, Wroclaw University of Science and Technology \\ W. Wyspianskiego street 27, 50-370 Wroclaw, Poland \\ krzysztof.tchon@pwr.edu.pl; joanna.ratajczak@pwr.edu.pl
}

\begin{abstract}
We address the motion planning problem in non-holonomic robotic systems using the tools of control theory. To this objective we associate with the robotic system a control-affine system. The derivative of the end-point map of this control system defines the Jacobian of the robotic system. Control functions at which the Jacobian is not surjective are referred to as singular configurations of the robotic system. As a description of these singularities we propose normal forms under feedback of the associated control system. On the basis of the Jacobian we introduce Jacobian motion planning algorithms. Special attention is paid to Lagrangian Jacobian algorithms. As an illustration of theoretical concepts we analyse normal forms, singularities, and motion planning for a free-floating space manipulator. A motion planning problem is solved by a Lagrangian Jacobian motion planning algorithm and, alternatively, by a sinusoidal control applied to the normal form. Results of computations show the performance of the two algorithms.
\end{abstract}

Keywords: non-holonomic system, affine Pfaffian constraints, control system, Jacobian, singularities, normal forms, motion planning, space manipulator

\section{Introduction}

The concept of a non-holonomic robotic system refers to robots whose motion is subject to a number of velocity constraints that are non-holonomic. Examples of such systems are widespread: they include the majority of mobile robots and mobile manipulators. Usually, the velocity constraints have Pfaffian form, either linear or affine. The former describes the kinematics of mobile robots, the latter characterizes the dynamics of free-floating space manipulators with non-zero conserved momenta. Contrary to the holonomic robotic systems (robotic manipulators), conceptual tools for the analysis of non-holonomic robotic systems have not yet been established in a commonly accepted form. To some extent this results from the very nature of non-holonomic systems; for example, the concept of singular configurations for robotic manipulators is unique and well known, mathematically covered by singularity theory of maps, while for non-holonomic robotic systems there are two diverse concepts derived from geometric control theory: control distribution (posture) or control function (configuration) singularities, not to mention some ad hoc constructions. The main objective of this paper is to show that an intertwining of robotic and control theoretical concepts can lay systematic foundations for a theory of non-holonomic robotic systems. Basic references inspiring the undertaking of this objective are: the homotopy method, introduced into robotics by Sussmann [1] and developed further in [2-4], theory of geometric and optimal control [5-8], theory of feedback invariants [9], sub-Riemannian geometry [10], theory of linear time dependent systems [11], and last but not least, our own work devoted to the Endogenous Configuration Space Approach [12].

We believe that any theory of non-holonomic robotic systems should as far as possible be consistent with the existing theory of holonomic robotic systems. To this objective we use the framework of control theory in the following way. Having associated with Pfaffian constraints a control system, we focus on the end-point map of this system, a standard concept in geometric control and sub-Riemannian geometry, that transforms control functions into system's states. Given the end-point map, the Jacobian of the robotic system is understood as the derivative of this map with respect to the control function. The computation of the Jacobian is done by the linear approximation of the associated control system along a fixed control-state trajectory (the associated variational system). It follows that for a given control function the Jacobian is a linear transformation of the space of control functions into the state space. Control functions at which this operator is a surjection (has full rank) are called regular configurations of the non-holonomic robotic system, otherwise the configurations are singular. When dealing with singularities we use the fact that singularities of the end point map are identical with the singular controls of the associated control system. Moreover, at regular controls the associated control system is locally controllable. 
This controllability condition allows then to check regularity of configurations. It has been shown that singular controls are preserved by feedback. For this reason, in order to characterize singularities of a non-holonomic robotic system we propose to exploit normal forms under feedback of the associated control system. By the normal form we mean a control system of a very simple form, feedback equivalent to the associated control system. A fundamental problem addressed in robotic systems is the motion planning problem. Referring to the associated control system this problem amounts to determining a control function that drives the state at a prescribed time to a prescribed point. Similarly as for holonomic robots, the motion planning problem in non-holonomic robotic systems can be solved by Jacobian motion planning algorithms. A derivation of these algorithms relies on the method of homotopy, that provides a solution to the motion planning problem as the limit of a curve in the control functions solving a functional differential equation governed by a right Jacobian inverse. This inverse is often obtained by reference to an optimal control problem addressed in the associated variational system. If the objective function of this problem is of Lagrange type, the inverse is referred to as the Lagrangian Jacobian Inverse. A general form of his inverse has been derived by means of the Pontryagin's Maximum Principle. Thanks to a considerable freedom in choosing the Lagrangian objective function, the Lagrangian Jacobian Inverse is capable of accommodating quite many design requirements imposed on the resulting motion planning algorithm. For illustration of our approach we have chosen the dynamics model of a space manipulator designed recently in the Space Research Centre of the Polish Academy of Sciences [13], [14], called the SRC manipulator. The SRC manipulator is a free-floating manipulator composed of a planar mobile base carrying on board a 2 DOF planar manipulator, built to be used in experiments with capturing Space debris. The floating of the SRC manipulator preserves the linear and angular momenta, so its centre of mass moves uniformly and rectilinearly. The angular motion of the manipulator fulfils the affine Pfaffian constraints, and can be represented by a control-affine associated system whose controls are velocities of the on board manipulator's joints. For this system we define Jacobian and singularities, introduce normal forms under feedback, and solve a motion planning problem by means of a specific Lagrangian Jacobian algorithm that distinguishes by a minimum energy of the variational trajectory [15]. As a counterpoint, we use a normal form and solve the motion planning problem using the sinusoid control algorithm [16]. Computer simulations demonstrate the performance of these algorithms.

The organization of this paper is the following. Section 2 presents basic concepts, concluding with the Jacobian. Singularities, normal forms, Lagrangian Jacobian Inverse, and the motion planning are studied in section 3. In section 4 we apply the theoretical concepts to the SRC manipulator, and display results of computations. Section 5 concludes the paper.

\section{Basic Concepts}

As a point of departure we take a robotic system described by generalized coordinates $q \in R^{n}$ and velocities $\dot{q} \in$ $R^{n}$, whose motion is subject to a number $l<n$ of non-holonomic phase constraints expressed in the affine Pfaffian form

$$
\boldsymbol{A}(q) \dot{q}=b(q)
$$

Hereabout $\boldsymbol{A}(q)$ denotes a rank $l,(l \times n)$-matrix-valued function, and $b(q) \in R^{l}$; both functions depend smoothly on $q$. It follows that constraints (1) can be expressed in the form of a control-affine system

$$
\dot{q}=f(q)+\sum_{i=1}^{m} g_{i}(q) u_{i}=f(q)+G(q) u,
$$

where the state variable $q \in R^{n}$ and the control variable $u \in R^{m}, m=n-l$. If $\boldsymbol{A}^{\#}(q)$ denotes a right inverse of matrix $\boldsymbol{A}(q)$ (i. e. $\boldsymbol{A}(q) \boldsymbol{A}^{\#}(q)=I_{l}, \quad I_{l}$ is the $l \times l$ unit matrix) then in (2) the drift vector field $f(q)=\boldsymbol{A}^{\#}(q) b(q)$. Control vector fields $g_{1}(q), g_{2}(q), \ldots, g_{m}(q)$ span the null space $\operatorname{Ker} A(q)$ at every point $q$. One may notice that system (2) is not defined uniquely, namely, if we use a feedback transforming $u$ to $u=\alpha(q)+\beta(q) v, \alpha(q)$ an arbitrary function, matrix $\beta(q)$ of full rank $m$, then for 


$$
\tilde{f}(q)=f(q)+G(q) \alpha(q), \quad \tilde{G}(q)=G(q) \beta(q)
$$

the control-affine system $\dot{q}=\tilde{f}(q)+\tilde{G}(q) v$ also represents constraints (1). Control-affine system (2) will be referred to as the associated control system to the non-holonomic robotic system. The associated system is controlled over a time horizon $T>0$. Its admissible control functions $U=L_{m}^{2}[0, T]$ are Lebesgue square integrable $R^{m}$-valued functions of time, defined on $[0, T]$. For an initial state $q_{0}$ and an admissible control function $u(\cdot), q(t)=\varphi_{q_{0}, t}(u(\cdot))$ denotes the state trajectory of (2). The transformation of an initial state and of a control function into the state variable at the end of the time horizon

$$
H_{q_{0}, T}(u(\cdot))=\varphi_{q_{0}, T}(u(\cdot))
$$

is called the end-point map of the associated control system. Relying on an analogy between holonomic and non-holonomic systems we treat the control function as a configuration of the non-holonomic robotic system. The derivative of (4) with respect to the control function defines the Jacobian of the robotic system,

$$
D H_{q_{0}, T}(u(\cdot))=J_{q_{0}, T}(u(\cdot))
$$

It is a standard fact of control theory [17] that the Jacobian is computed by the linear approximation

$$
\dot{\xi}=A(t) \xi+B(t) v
$$

to the associated control system, whose state variable $\xi \in R^{n}$ and control variable $v \in R^{m}$. For a control $u(t)$ in (2) and $q(t)$ being the corresponding trajectory, matrices of the linear approximation are defined as

$$
A(t)=\frac{\partial(f(q)+G(q(t)) u(t))}{\partial q}, \quad B(t)=G(q(t))
$$

By computing the state of (6) at time $T$ for a given control variation $v(\cdot) \in U$ we get the following formula for the Jacobian

$$
J_{q_{0}, T}(u(\cdot)) v(\cdot)=q(T)=\int_{0}^{T} \Phi(T, t) B(t) v(t) d t
$$

where the fundamental matrix satisfies the differential equation

$$
\frac{\partial \Phi(t, s)}{\partial t}=A(t) \Phi(t, s), \quad \Phi(s, s)=I_{n}
$$

\section{Motion Planning}

With reference to the associated control system, the motion planning problem for a non-holonomic robotic system with given desired state $q_{d}$ consists in determinig a control $u_{d}(t)$ such that $H_{q_{0}, T}\left(u_{d}(\cdot)\right)=q_{d}$. It follows that solving the motion planning problem involves the inversion of the end-point map. This inversion can be done by Jacobian motion planning algorithms relying on a right inverse $J_{q_{0}, T}^{\#}(u(\cdot))$ of the Jacobian, i.e. an operator having the property $J_{q_{0}, T}(u(\cdot)) J_{q_{0}, T}^{\#}(u(\cdot))=$ $I_{n}$. A general derivation of a Jacobian algorithm consists in finding a solution for a curve $u_{\vartheta}(\cdot)$ of control functions parametrized by $\vartheta \in R$, of the differential equation

$$
\frac{d u_{\vartheta}(\cdot)}{d \vartheta}=-\gamma J_{q_{0}, T}^{\#}\left(u_{\vartheta}(\cdot)\right)\left(H_{q_{0}, T}\left(u_{\vartheta}(\cdot)\right)-q_{d}\right)
$$


initialized at some $u_{\vartheta=0}(\cdot)=u_{0}(\cdot)$, where $\gamma>0$ denotes the error decay rate. Indeed, if $e(\vartheta)=H_{q_{0}, T}\left(u_{\vartheta}(\cdot)\right)-q_{d}$ is the planning error corresponding to $u_{\vartheta}(t)$ then the differentiation of the error with respect to the parameter results in

$$
\frac{d e(\vartheta)}{d \vartheta}=J_{q_{0}, T}\left(u_{\vartheta}(\cdot)\right) \frac{d u_{\vartheta}(\cdot)}{d \vartheta}=-\gamma e(\vartheta)
$$

The error decays exponentially providing in the limit a solution to the motion planning problem, $u_{d}(t)=\lim _{\vartheta \rightarrow+\infty} u_{\vartheta}(t)$.

\subsection{Singularities}

By definition, a right inverse of the Jacobian exists on condition that the Jacobian, regarded as a map from the admissible control functions to the state space, is surjective, i.e. the end-point map is a submersion. A control function $u(\cdot) \in U$ at which the Jacobian gets surjective is called a regular configuration of the robotic system, otherwise the configuration is referred to as singular. It is well known that the singular configuration coincides with the singular control studied in optimal control theory. A necessary and sufficient condition for regularity of $u(\cdot)$ is the full rank of the Gram matrix

$$
\boldsymbol{G}_{q_{0}, T}(u(\cdot))=\int_{0}^{T} \Phi(T, t) B(t) B^{T}(t) \Phi^{T}(T, t) d t
$$

\subsection{Normal Forms}

A control-affine system of a simple form that is feedback equivalent to the control system associated with the robotic system will be called a normal form of the robotic system. We recall that two control-affine systems $\Sigma$ and $\tilde{\Sigma}$

$$
\Sigma: \dot{q}=f(q)+G(q) u, \quad \tilde{\Sigma}: \dot{\tilde{q}}=\tilde{f}(\tilde{q})+\tilde{G}(\tilde{q}) \tilde{u}
$$

with state variables $q, \tilde{q} \in R^{n}$ and inputs $u, \tilde{u} \in R^{m}$ are feedback equivalent if there exist a change of coordinates $\tilde{q}=$ $\varphi(q)$ and an input transformation $u=\alpha(q)+\beta(q) \tilde{u}$, defined by an $R^{m}$-valued function $\alpha(q)$ and an invertible $m \times$ $m$ matrix $\beta(q)$, both smoothly dependent on $q$, such that

$$
\frac{\partial \varphi(q)}{\partial q}(f(q)+G(q) \alpha(q))=\tilde{f}(\varphi(q)), \quad \frac{\partial \varphi(q)}{\partial q} G(q) \beta(q)=\tilde{G}(\varphi(q))
$$

Thanks to the simplicity of the normal form, checking its properties is much easier than of the original system, provided that the properties under examination have been preserved by the feedback. Since singular configurations are preserved by feedback [18], it is possible to describe singularities of robotic systems by their normal forms. Furthermore, if the feedback transformations are known explicitly the normal form may also contribute to designing a motion planning algorithm, [19].

\subsection{Lagrangian Jacobian Motion Planning}

There exist many specific right inverses of the Jacobian. In the recent paper [15] we have introduced the Lagrangian Jacobian inverse defined as the solution to the Lagrange-type optimal control problem addressed in associated variational system (6), (7):

$$
\min _{v(\cdot)} \frac{1}{2} \int_{0}^{T}\left(\begin{array}{l}
\xi(t) \\
v(t)
\end{array}\right)^{T}\left[\begin{array}{cc}
Q(t) & S(t) \\
S^{T}(t) & R(t)
\end{array}\right]\left(\begin{array}{l}
\xi(t) \\
v(t)
\end{array}\right) d t
$$


with equality constraint $J_{q_{0}, T}(u(\cdot)) v(\cdot)=\xi(T)=w, w \in R^{n}$. For well-definiteness the matrices appearing in the Lagrangian objective function need to fulfil conditions $Q(t)=Q^{T}(t) \geqslant 0, R(t)=R^{T}(t)>0, Q(t)-S(t) R^{-1}(t) S^{T}(t) \geqslant$ 0 . The following form of the Lagrangian Jacobian inverse $J_{q_{0}, T}^{L J \#}$ has been found using the Pontryagin's Maximum Principle

$$
\left(J_{q_{0}, T}^{L J \#}(u(\cdot)) w\right)(t)=R^{-1}(t)\left(B^{T}(t) \phi_{22}(t)-S^{T}(t) \phi_{12}(t)\right) \phi_{12}^{-1}(T) w
$$

where matrix $\phi(t)=\left[\phi_{i j}(t)\right], i, j=1,2$, solves the differential equation

$$
\dot{\phi}(t)=\left[\begin{array}{cc}
A(t)-B(t) R^{-1}(t) S^{T}(t) & B(t) R^{-1}(t) B^{T}(t) \\
Q(t)-S(t) R^{-1}(t) S^{T}(t) & -A^{T}(t)+S(t) R^{-1}(t) B^{T}(t)
\end{array}\right] \phi(t),
$$

with initial condition $\phi_{i j}(0)=\delta_{i j} I_{n}, i, j=1,2$, and $\delta_{i j}$ denoting the Kronecker function. Having plugged $J_{q_{0}, T}^{L J \#}(u(\cdot))$ into (10) we obtain the Lagrangian Jacobian motion planning algorithm

$$
\frac{d u_{\vartheta}(\cdot)}{d \vartheta}=-\gamma J_{q_{0}, T}^{L J I \#}\left(u_{\vartheta}(\cdot)\right)\left(H_{q_{0}, T}\left(u_{\vartheta}(\cdot)\right)-q_{d}\right)
$$

A specific instance of the Lagrangian Jacobian motion planning algorithm is obtained after choosing $Q(t)=A^{T}(t) A(t)$, $S(t)=A^{T}(t) B(t), R(t)=B^{T}(t) B(t)$. Notice that with this choice we get the identity $Q(t)-S(t) R^{-1}(t) S^{T}(t)=0$, so the matrix of the quadratic form in the objective function (15) becomes singular. The Lagrangian Jacobian Inverse based on this specific choice will be denoted by $J_{q_{0}, T}^{S L I \#}$ ( $S$ means specific). It has been shown that this inverse minimizes the energy contained in the variation of the state trajectory of the associated control system [15]. We shall use it in example computations.

\section{Exemplification}

The concepts introduced in the previous sections will now be applied to the model of dynamics of a free-floating space manipulator designed in the Space Research Centre (SRC) of the Polish Academy of Science [13], see Figure 1. The SRC manipulator is composed of a planar base carrying on board a 2 DOF planar manipulator. Geometric and dynamic parameters of the SRC manipulator have been specified in [14].
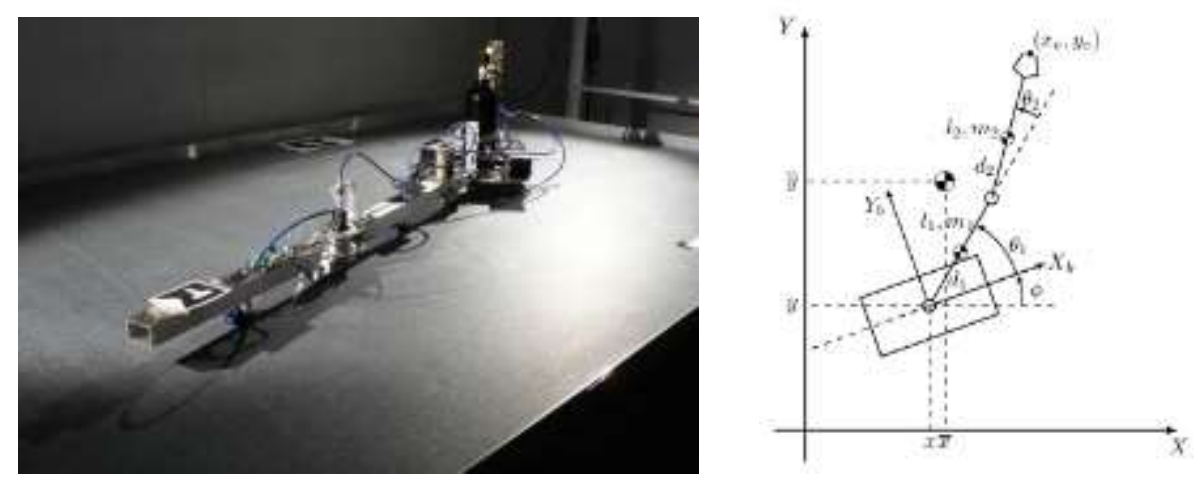

Fig. 1: SRC space manipulator. 
The Lagrangian Jacobian motion planning for this manipulator has been studied earlier in [15] under assumption that the conserved angular momentum is zero, so the associated control system has been driftless. Here we allow for a non-zero angular momentum.

\subsection{Equations of Motion}

Having chosen coordinates $q=\left(\bar{x}, \bar{y}, \phi, \theta_{1}, \theta_{2}\right)$, where $\bar{x}, \bar{y}$ refer to the position of the centre of mass of the whole space manipulator, and $\phi, \theta_{1}, \theta_{2}$ denote, respectively, the orientation of the base and the joint positions of the on-board manipulator, the Lagrangian of the SRC manipulator can be computed as

$$
L(q, \dot{q})=\frac{1}{2} m_{c}\left(\dot{\bar{x}}^{2}+\dot{\bar{y}}^{2}\right)+\frac{1}{2} I_{b} \dot{\phi}^{2}+\frac{1}{2} D\left(\dot{\phi}+\dot{\theta}_{1}\right)^{2}+\frac{1}{2} E\left(\dot{\phi}+\dot{\theta}_{12}\right)^{2}+F \cos \theta_{2}\left(\dot{\phi}+\dot{\theta}_{1}\right)\left(\dot{\phi}+\dot{\theta}_{12}\right) .
$$

Hereabout $m_{c}=M+m_{1}+m_{2}$ is the total mass of the base and of the links, $I_{b}$ denotes the moment of inertia of the base, $\theta_{12}=\theta_{1}+\theta_{2}$, and

$$
\begin{gathered}
D=\frac{1}{m_{c}}\left(m_{1} m_{2}\left(l_{1}-d_{1}\right)^{2}+M\left(m_{1} d_{1}^{2}+m_{2} l_{1}^{2}\right)\right), \quad E=\frac{1}{m_{c}}\left(M+m_{1}\right) m_{2} d_{2}^{2}, \\
F=\frac{1}{m_{c}}\left(m_{1} m_{2}\left(l_{1}-d_{1}\right) d_{2}+M m_{2} l_{1} d_{2}\right) .
\end{gathered}
$$

\subsection{Affine Pfaffian Constraints}

The motion equations resulting from Lagrangian (19) obey the conservation law of linear and angular momenta. This implies that the centre of mass of the space manipulator will move in Space uniformly and rectilinearly. Further, the conservation of the angular momentum yields the following affine Pfaffian constraint

$$
K\left(\theta_{2}\right) \dot{\phi}+M\left(\theta_{2}\right) \dot{\theta}_{1}+N\left(\theta_{2}\right) \dot{\theta}_{2}=p,
$$

where $K\left(\theta_{2}\right)=I_{b}+D+E+2 F \cos \theta_{2}, M\left(\theta_{2}\right)=D+E+F \cos \theta_{2}, N\left(\theta_{2}\right)=E+F \cos \theta_{2}$, and the constant $p$ denotes the conserved angular momentum. It is assumed that the space manipulator is controlled by the rotation velocities of the joints. Now, let us introduce a global change of coordinates $\left(\phi, \theta_{1}, \theta_{2}\right) \rightarrow\left(\alpha, \beta, \theta_{2}\right)$ defined as

$$
\alpha=\left(I_{b}+D+E\right) \phi+(D+E) \theta_{1}+E \theta_{2}+F \sin \theta_{2}, \quad \beta=\phi+\theta_{1}, \quad \theta_{2}=\theta_{2}
$$

In new coordinates the control system associated with constraint (21) takes the following form:

$$
\dot{\alpha}=p-2 F \cos \theta_{2} u_{1}, \quad \dot{\beta}=u_{1}, \quad \dot{\theta}_{2}=u_{2}
$$

Observe that the original orientation angle $\phi$ and the joint position $\theta_{1}$ can be retained easily from (22).

\subsection{Normal Forms and Singularities}

Let us distinguish the set $S_{2}=\left\{\left(\phi, \theta_{1}, \theta_{2}\right) \mid \sin \theta_{2}=0\right\}$ of kinematic singularities of the on-board manipulator, and assume that $p \neq 0$. We recall that a control $u(t)$ in (2) is referred to as singular if the Gram matrix

$$
\boldsymbol{G}_{q_{0}, T}(u(\cdot))=\int_{0}^{T} \Phi(T, t) B(t) B^{T}(t) \Phi^{T}(T, t) d t
$$

is rank deficient. It has been shown in [18] that the dynamics of the SRC manipulator can be given by feedback either of the two following normal forms.

- $\quad$ Outside $S_{2}$ the normal form is Pfaff-Darboux [20], 


$$
\dot{z}_{1}=1+z_{2} v_{2}, \quad \dot{z}_{2}=v_{1}, \quad \dot{z}_{3}=v_{2},
$$

and the singular controls are trivial: $v_{1}(t)=v_{2}(t)=0$.

- $\quad$ Inside $S_{2}$ the normal form is Martinet [6],

$$
\dot{z}_{1}=1+z_{2}^{2} v_{2}, \quad \dot{z}_{2}=v_{1}, \quad \dot{z}_{3}=v_{2},
$$

whose singular controls are $v_{1}(t)$ - arbitrary and $v_{2}(t)=0$. In this way the normal forms describe singularities of the SCR manipulator. Furthermore, since normal form (25) resembles the chained form, the motion planning problem in this normal form can be solved by the simple sinusoid control, see [16] and [19]. Fused with the feedback

$$
z_{1}=\frac{\alpha}{p}, \quad z_{2}=2 F c_{2}, \quad z_{3}=-\frac{\beta}{p}, \quad u_{1}=-p v_{2}, \quad u_{2}=-\frac{1}{2 F \sin \theta_{2}} v_{1}
$$

that establishes the equivalence between (23) and (25), the sinusoid control can provide an alternative solution to the motion planning problem in associated system (2).

\subsection{Motion Planning}

We study a motion planning problem for the SRC manipulator floating with the conserved angular momentum $p=$ 0.02 , consisting in reaching the desired point $q_{d}=\left(-\frac{\pi}{10}, \frac{\pi}{6}, \frac{\pi}{2}\right)$ from $q_{0}=\left(0,0, \frac{\pi}{8}\right)$ in time $T=20$. Results are displayed in figures 2 and 3, for two motion planning algorithms: $J_{q_{0}, T}^{S L J \#}$ and the sinusoid control $(N F+S I N)$, applied to normal form (25) and transformed back to the associated control system by (22). The figures show the orientation of the base, the path of the end effector with respect to the $\left(X_{b}, Y_{b}\right)$ frame, where $x_{e}=l_{1} \cos \theta_{1}+l_{2} \cos \theta_{12}, y_{e}=l_{1} \sin \theta_{1}+l_{2} \sin \theta_{12}$, and the control energy spent at consecutive steps of the algorithms (there are no steps in the sinusoid control). The sinusoid algorithm distinguishes by simplicity and efficiency. On the other hand, the Lagrangian Jacobian algorithm, although much more complex, generates smaller motions and saves control energy, what may be desirable in Space applications.
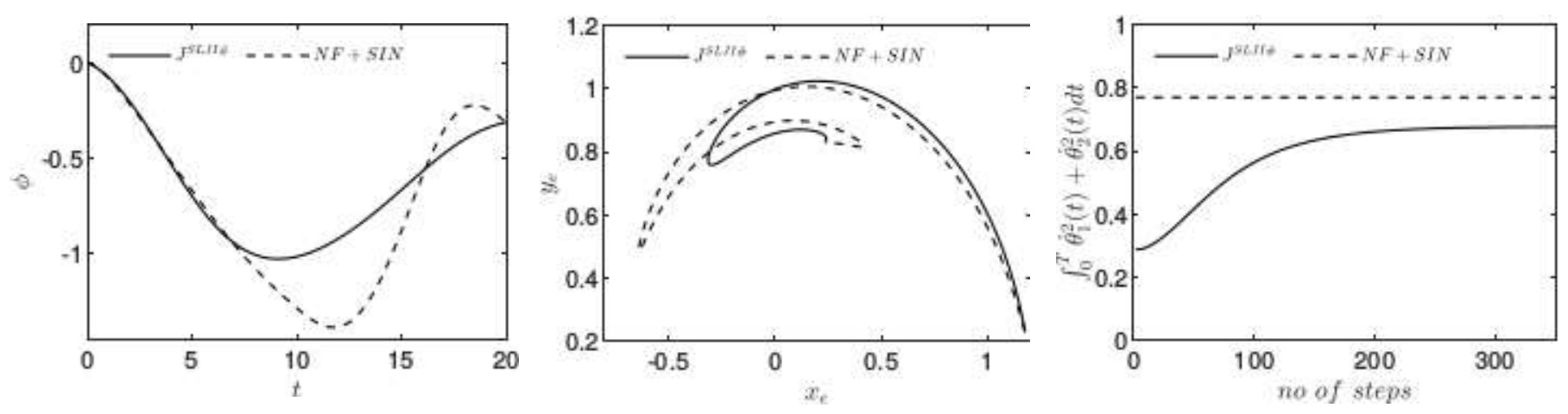

Fig. 2: Results of computations.

\section{Conclusion}

Relying on instruments of the modern control theory this paper makes a contribution to the conceptual foundations of the theory of non-holonomic robotic systems. Special attention has been paid to the Lagrangian Jacobian motion planning, and to the role of the feedback equivalence and of the feedback normal forms in describing singularities. Usefulness of the proposed concepts has been demonstrated by an application to a space manipulator. In this context an energy saving Lagrangian Jacobian motion planning algorithm has been exploited, and two normal forms established and used as a tool for describing singularities as well as for designing a motion planning algorithm based on sinusoid control. Further research 
will include experiments with the SRC manipulator as well as a study of normal forms and Jacobian motion planning of free-floating space manipulators equipped with more redundancy on board.

\section{Acknowledgements}

This research was supported by the Wroclaw University of Science and Technology under a research project No 0401/0019/18 ( $1^{\text {st }}$ author), and by the National Science Centre under a project No 2015/17/B/ST7/03995 ( ${ }^{\text {nd }}$ author).

\section{References}

[1] H. J. Sussmann, "A continuation method for nonholonomic path finding problems," in Proc. 32nd IEEE CDC, pp. 2718-2723, 1993.

[2] A. W. Divelbiss, S, Seereeram and J. T. Wen, "Kinematic path planning for robots with holonomic and nonholonomic constraints," in: Essays on Mathematical Robotics, J. Baillieul, S. S. Sastry and H. J. Susmann, Eds. Springer-Verlag, 1998, pp. 127-150.

[3] Y. Chitour, "A continuation method for motion planning problems," ESAIM, vol. 12, no. 1, pp. 139-168, 2006.

[4] F. Alouges, Y. Chitour and R. Long, "A motion planning algorithm for the rolling-body problem," IEEE Trans. Robotics, vol. 26, no. 5, pp. 827-836, 2010.

[5] C. D. Johnson and J. E. Gibson, "Singular solutions in problems of optimal control," IEEE Trans. Autom. Contr., vol. 8, no 1, pp. 4-15, 1963.

[6] B. Bonnard and M. Chyba, Singular Trajectories and Their Role in Control Theory, Springer, Paris, 2003.

[7] Y. Chitour, F. Jean and E. Trélat, "Singular trajectories of control-affine systems," SIAM J. Contr. Opt., vol. 47, no. 2, pp. 1078-1095, 2008.

[8] A. L'Affito and W. M. Haddad, "Abnormal optimal trajectory planning of multi-body systems in the presence of holonomic and nonholonomic constraints," J. Intell. Robotic Syst., vol. 89, no 1-2, pp. 51-67, 2018.

[9] B. Jakubczyk, "Critical Hamiltonians and feedback invariants," in Geometry of Feedback and Optimal Control, B. Jakubczyk and W. Respondek Eds. Marcel Dekker, New York, 1998, pp. 218-256.

[10] R. A. Montgomery, A Tour of Subriemannian Geometries, Their Geodesics and Applications, American Mathematical Society, 2002.

[11] W. J. Rugh, Linear System Theory, Prentice Hall, Englewood Cliff, 1993.

[12] K. Tchon, "Endogenous Configuration Space Approach: An Intersection of Robotics and Control Theory," in Nonlinear Systems, N. van de Wouv, E. Lefeber and I. L. Arteaga Eds., Springer, 2017, pp. 209-234.

[13] T. Rybus and K. Seweryn,"Planar air-bearing microgravity simulators: review of applications, existing solutions and design parameters," Acta Astronaut., vol. 120, pp. 239-259, 2016.

[14] T. Rybus, K. Seweryn and J. Sasiadek, "Application of predictive control for manipulator mounted on a satellite," Archives of Control Sciences, vol. 28, no. 1, pp. 105-118, 2018.

[15] K. Tchon and J. Ratajczak, "General Lagrange-type Jacobian inverse for nonholonomic robotic systems," IEEE Trans. Robotics, vol. 34, no. 1, pp. 256-263, 2018.

[16] R. Murray, Z. Li and S. S. Sastry, A Mathematical Introduction to Robotic Manipulation, CRC Press, 1994.

[17] E. D. Sontag, Mathematical Control Theory, Springer, New York, 1990.

[18] K. Tchon, W. Respondek and J. Ratajczak, "Normal forms and configuration singularities of a space manipulator," $J$. Intell. Robotic Syst., vol. 93, pp. 621-634, 2019.

[19] K. Tchon, J. Ratajczak and J. Jakubiak, "Normal forms of robotic systems with affine Pfaffian constraints: A case study," in Advances in Robot Kinematics 2018, J. Lenarcic and V. Parenti-Castelli, Eds. Springer, 2019, pp. $250-257$.

[20] R. L. Bryant, et al., Exterior Differential Systems, Springer, New York, 1991. 
127-9 